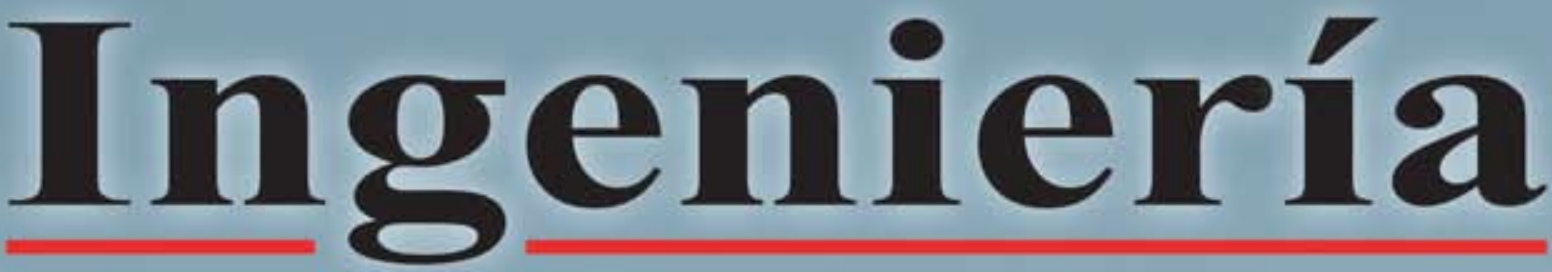

ENERO/DICIEMBRE 2004 - VOLUMEN 14 - N¹ y 2

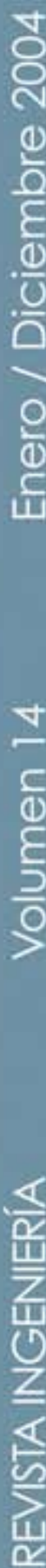
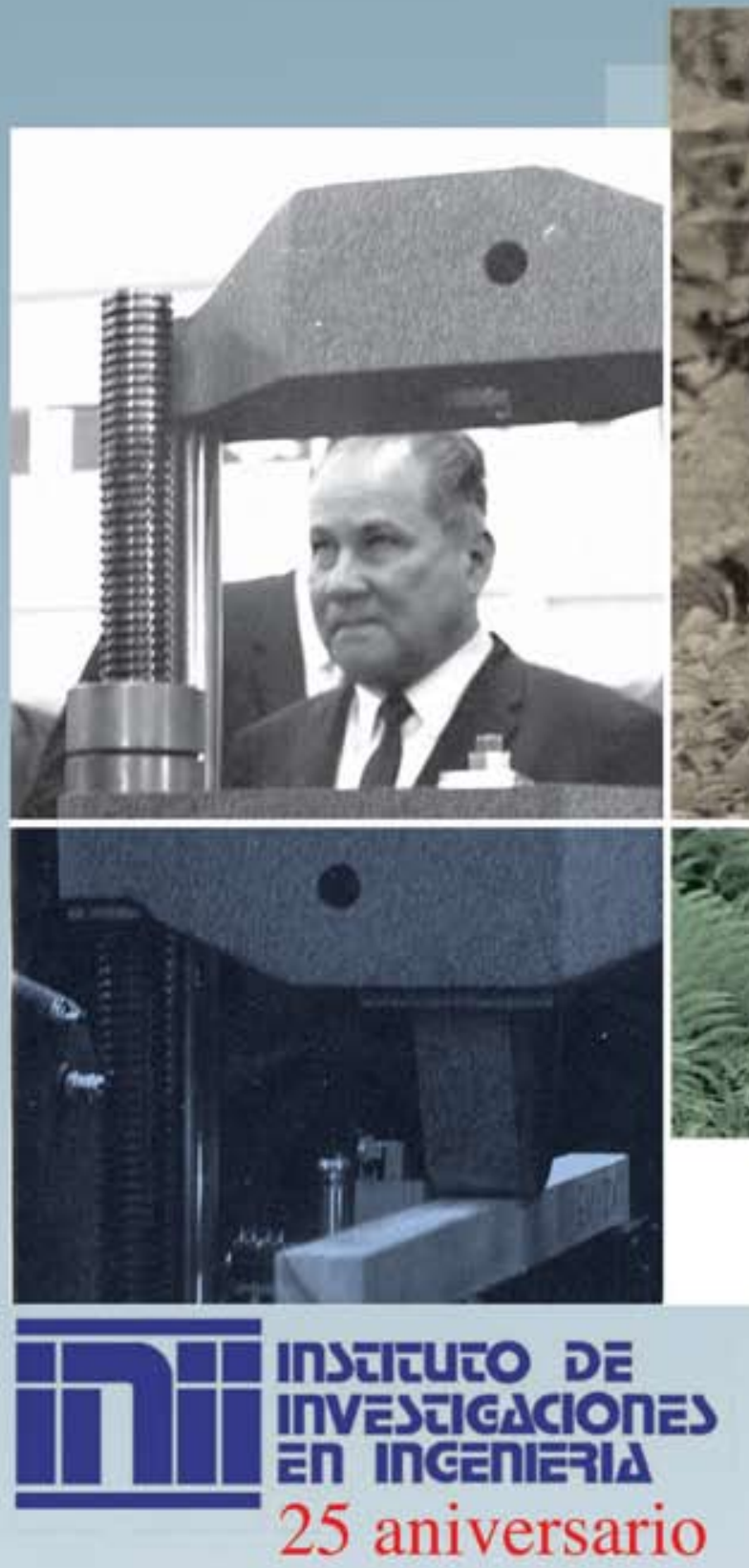

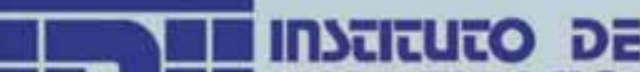
T W Investcscines हn Incenlei|d 25 aniversario

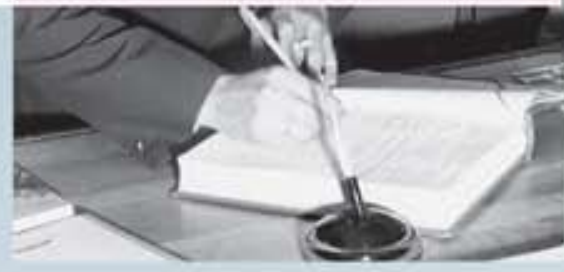




\title{
APLICACIÓN DEL CONTROL ÓPTIMO LINEAL A LA ESTABILIDAD DINÁMICA DE UN SISTEMA DE POTENCIA
}

\author{
Eddie A. Araya Padilla
}

\begin{abstract}
Resumen
Este trabajo presenta la aplicación del control óptimo lineal para mejorar la estabilidad dinámica de un sistema de potencia, mediante el amortiguamiento de oscilaciones de potencia menores a $1 \mathrm{~Hz}$. Estas oscilaciones establecen un límite a la operación en régimen permanente y físicamente, representan los modos naturales de oscilación de áreas eléctricas, constituidas por generadores dinámicamente coherentes.

La metodología propuesta se aplica al sistema de potencia centroamericano, porque presenta una topología longitudinal y se encuentra débilmente mallado. El análisis se efectúa para diferentes condiciones de operación y los resultados comprueban la efectividad de la técnica utilizada.
\end{abstract}

Palabras clave: control óptimo lineal, estabilidad dinámica, amortiguamiento, sistema de potencia, modos naturales de oscilación.

\begin{abstract}
This paper presents the application of optimal linear control to improve the dynamic stability of a power system, by damping power oscillations of up to $1 \mathrm{~Hz}$. These oscillations limit steady state operation and physically represent the natural modes of oscillation of electric areas, served by dynamically coherent generators.

The proposed methodology is applies to the Central American electrical grid because it is a longitudinal system and it is weakly meshed. The analysis is performed under different operation conditions and the results confirm the effectiveness of the technique.
\end{abstract}

Keywords: optimal linear control, dynamic stability, damping, power system, natural modes of oscillation

Recibido 01-III-04 • Aceptado 14-X-04

\section{INTRODUCCIÓN}

En los sistemas de potencia débilmente mallados, las líneas de transmisión, por su elevada reactancia inductiva, operan con un reducido margen de estabilidad en régimen permanente, a menos que se instalen en ellas dispositivos de compensación. Si, además, dentro de estos sistemas se tienen subsistemas de muy diferente potencia instalada, los cuales están vinculados por líneas de transmisión de reducida capacidad (menor que la potencia instalada del subsistema más pequeño) y se transmiten altos niveles de potencia activa, entonces surgen problemas de oscilaciones de potencia (OP) en los enlaces de alta tensión. Este problema se incrementa por las reacciones de los reguladores de velocidad ante las variaciones de la carga en los subsistemas vinculados. Las OP ocurren con cierta frecuencia en sistemas reales como el centroamericano (Quesada, 1986; Sancho, 1998), el argentino (Molina, 1997) y el mejicano (Messina, 1995), entre otros.

La única forma efectiva para amortiguar las OP es alterar las características dinámicas inherentes del sistema. A la fecha, muchas investigaciones se han llevado a cabo para disminuir las OP en los sistemas de potencia débilmente mallados. Entre los métodos de solución propuestos se encuentran los estabilizadores de sistemas de potencia PSS (PSS: Power System Stabilizer). 


\section{DISPOSITIVO PSS PARA AMORTIGUAR OP}

Los dispositivos PSS son localizados en los generadores que presentan problemas de amortiguamiento electromecánico. La idea básica del PSS es extender los límites de estabilidad mediante la modulación del sistema de excitación del generador, proporcionando amortiguamiento a las oscilaciones de los rotores.

Estos dispositivos se acoplan entre dos lazos de control, el de carga-frecuencia y el de tensiónpotencia reactiva. Su principio básico se muestra en la Figura 1 y consiste en tomar señales del lazo de control carga-frecuencia, tales como velocidad del eje de la máquina, frecuencia en las barras ac, potencia activa entregada a la red o potencia acelerante; para procesarlas e introducir su salida en el sistema de control de la tensión de excitación (AVR: automatic voltaje regulator) como una señal suplementaria. En este caso, en la ecuación diferencial de oscilación, además del amortiguamiento debido a los devanados amortiguadores, se introduce un amortiguamiento suplementario mediante la contribución del PSS, de forma tal que se mejora el amortiguamiento de las OP.

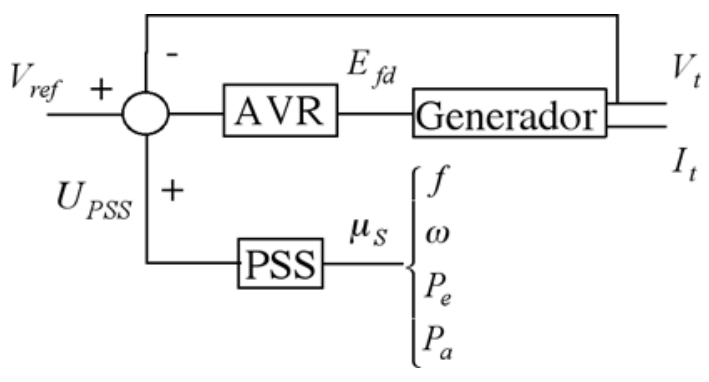

Figura 1. Esquema de vinculación entre el generador, el sistema de control de la tensión de excitación y el dispositivo PSS.

El dispositivo PSS consta por lo general de tres bloques:

- Un filtro con dos etapas para compensación de fase, que provee el adelanto de fase necesario para compensar el atraso de fase entre la entrada del sistema de excitación y el par eléctrico del generador.

- Un filtro wash-out que determina la frecuencia mínima a partir de la cual el PSS comenzará a aportar amortiguamiento.

- Una ganancia que delimita la cantidad de amortiguamiento introducido por este dispositivo.

Sin embargo, en este trabajo se pretende establecer el efecto del PSS a través de su salida, $U_{P S S}$, con el propósito de mejorar el amortiguamiento de la máquina sincrónica, sin modificar, en la medida de lo posible, la frecuencia del modo de oscilación. Una vez conocida esta información, $\mathrm{y}$ en una investigación posterior, la idea es determinar una función de transferencia $G_{P S S}(s)$, que cumpla con esas características dinámicas.

\section{LA ECUACIÓN DE OSCILACIÓN Y EL PAR ELECTROMAGNÉTICO}

La ecuación diferencial de movimiento de la máquina sincrónica, puede representarse mediante la siguiente expresión:

$$
M \ddot{\delta}+D \dot{\delta}=T_{m}-T_{e}
$$

Si la máquina sincrónica es sometida a una pequeña perturbación, la ecuación (1) puede linealizarse alrededor del punto de operación, obteniéndose:

$$
M \Delta \ddot{\delta}+D \Delta \dot{\delta}=\Delta T_{m}-\Delta T_{e}
$$

La característica de operación del regulador automático de velocidad, conduce a que aproximadamente durante $1,5 \mathrm{~s}$ a $2,0 \mathrm{~s}$ después de producida una perturbación, la potencia mecánica de la turbina permanece constante. Con base en esto, se considera que $\Delta T_{m}=0$, y en consecuencia, la máquina sincrónica solamente se ve sometida al par electromagnético, $\Delta T_{e}$.

El cambio que experimenta el par electromagnético, puede expresarse como la 
suma de los cambios que se producen en los momentos sincronizante y de amortiguamiento:

$\Delta T_{e}=T_{S} \cdot \Delta \delta+T_{D} \cdot \Delta \omega$

A partir de la expresión (3) y considerando el hecho de que $\Delta \omega=\Delta \dot{\delta}$, entonces la ecuación (2) se transforma en una ecuación diferencial homogénea:

$$
M \Delta \ddot{\delta}+\left(D+T_{D}\right) \Delta \dot{\delta}+T_{S} \Delta \delta=0
$$

cuya solución se satisface a través de los autovalores:

$$
\lambda_{1-2}=\sigma \pm j \omega
$$

siendo

$\sigma=-\frac{D+T_{D}}{2 M}:$ amortiguamiento

$\omega=\omega_{n} \sqrt{1-\xi^{2}}: \begin{aligned} & \text { frecuencia angular } \\ & \text { amortiguada }\end{aligned}$

$\omega_{n}=\sqrt{\frac{T_{S}}{M}} \quad:$ frecuencia angular natural

$\xi=\frac{1}{2} \frac{D+T_{D}}{\sqrt{T_{S} M}}:$ amortiguamiento relativo

De estas expresiones surge que:

- Un aumento en el coeficiente $T_{S}$ del par sincronizante, aumenta la frecuencia angular natural $\omega_{n}$,

- un aumento en el coeficiente $T_{\boldsymbol{D}}$ del par de amortiguamiento, incrementa el amortiguamiento relativo $\xi$,

- un aumento en la constante $M$ disminuye la frecuencia angular natural $\omega_{\mathrm{n}} \mathrm{y}$ el amortiguamiento relativo $\xi$.

\section{EFECTO DE $U_{P S S}$ AL AMORTIGUAMIENTO DEL SISTEMA}

Considérese un sistema controlable invariante en el tiempo modelado en variables de estado como

$\Delta \dot{X}=A \Delta X+B \Delta U$

donde $X$ y $U$ son el vector de estado y el de control respectivamente. $A$ y $B$ son matrices constantes de dimensiones apropiadas.

La teoría del control óptimo lineal (Ogata, 1993) establece que el vector de control se obtiene de la expresión

$\Delta U=-F \Delta X$

donde $F$ es la matriz del lazo de realimentación de (6), la cual se calcula con el criterio de minimizar el índice cuadrático de funcionamiento definido por:

$J=\frac{1}{2} \int_{0}^{\infty}\left(X^{T} Q X+U^{T} R U\right) d t$

Este índice representa el gasto de energía de las funciones del tiempo $x_{i}(t)$ y $u_{j}(t)$ para $i=1$, $2, \ldots n$ y $j=1,2, \ldots m$. Las matrices $Q$ y $R$, que en principio son desconocidas, se denominan matrices de peso.

Reemplazando (7) en (6) surge la expresión para el sistema operando a lazo cerrado:

$\Delta \dot{X}=(A-B F) \Delta X$

Los autovalores de la matriz A-BF, denotados por $\Lambda(\mathrm{A}-\mathrm{BF})$, deberán localizarse en el semiplano izquierdo del plano $s$ para que el sistema sea estable.

La teoría del control óptimo lineal (Ogata, 1993) muestra también que si la ganancia $F$ del lazo de realimentación (7) surge de la siguiente expresión

$F=R^{-1} B^{T} P$ 
en donde $\mathrm{P}$ es una matriz simétrica definida positiva, que se obtiene resolviendo la ecuación matricial algebraica de Riccati (Ogata, 1993; Laub, 1979) presentada a continuación

$$
A^{T} P+P A-P B R^{-1} B^{T} P+Q=0
$$

entonces, efectivamente todos los autovalores de la matriz (A-BF) se ubican en el semiplano izquierdo del plano complejo. Este procedimiento, en la mayoría de los casos mantiene prácticamente inalteradas las frecuencias propias de oscilación del sistema.

Para calcular la matriz incógnita $P$ se conocen en (11) las matrices, $A, A^{T}, B$ y $B^{T}$ de (6) y deben suponerse valores para los elementos de las matrices $Q$ y $R$.

La acción de coordinación de las señales de control $u_{j}$ surge de la expresión (7). En efecto, observando esta expresión, cada señal de control $u_{j}$ es obtenida combinando información del estado de todo el sistema (vector $\Delta X$ ) mediante la correspondiente fila de la matriz $F$.

En la práctica del diseño de los sistemas óptimos lineales, la matriz $F$ se calcula aproximadamente asignando valores a las matrices de peso acorde al razonamiento físico, es decir, por prueba y error hasta lograr los efectos deseados. Para el caso de sistemas grandes y sobre todo cuando no se conocen suficientemente las reacciones del sistema, resulta muy complicado asignar valores a todos los elementos de las matrices $Q$ y $R$. Por esta razón, es una práctica común elegir a estas matrices como matrices diagonales.

Si el comportamiento dinámico de un sistema, representado por (6) con autovalores $\lambda_{1}, \ldots, \lambda_{m}, \lambda_{m+1}, \ldots, \lambda_{n}$, es insatisfactorio a causa de que, por ejemplo, los $m$ autovalores $\lambda_{l}$ a $\lambda_{m}$ tienen partes reales positivos, entonces, el objetivo del control óptimo lineal, instrumentado mediante las ecuaciones (9), (10) y (11) es reubicar esos $m$ autovalores en el semiplano izquierdo del plano complejo, manteniendo prácticamente inalteradas las frecuencias naturales de oscilación del sistema. Los autovalores de la matriz A, que inicialmente estaban situados en el semiplano izquierdo del plano complejo, permanecen en ese semiplano, ya que este procedimiento afecta fundamentalmente a las partes reales de los autovalores de la matriz A, ubicados inicialmente en el semiplano derecho del plano complejo.

En las referencias (Moussa \& Yu, 1972; Yu \& Moussa, 1972) que emplean este método la matriz $R$ se toma como diagonal, e igual a la identidad y los elementos de la matriz $Q$ se determinan mediante un procedimiento iterativo de prueba y error para lograr en los autovalores que inicialmente eran débilmente amortiguados un desplazamiento de las partes reales hacia valores cada vez más negativos sin modificar significativamente las partes imaginarias.

También en este trabajo se toma a la matriz $R$ como la matriz identidad, con lo cual se asigna igual peso a las $m$ señales de control y se logra que cada una de ellas actúe en forma independiente. Para el tratamiento de la matriz $Q$ se sigue el camino propuesto en las referencias (Kawasaki \& Shimemura, 1983; Shieh et al., 1986).

Este camino consiste en no utilizar la matriz $Q$ y en reubicar los autovalores $\lambda_{l}$ a $\lambda_{m}$ del sistema de lazo abierto en el semiplano izquierdo del plano complejo y a una distancia $h_{l} \geq 0$ del origen de coordenadas (Figura 2a), manteniendo inalterados los autovalores $\lambda_{m+1}$ a $\lambda_{n}$ ubicados inicialmente en el semiplano izquierdo del plano complejo. El valor $h_{l}$ representa el grado deseado de estabilidad del sistema eléctrico. Para lograr esta reubicación de autovalores, la matriz $F$ del lazo de realimentación (10) toma ahora el valor

$\widetilde{F}=R^{-1} B^{T} \widetilde{P}$

con lo cual la matriz del sistema a lazo cerrado (9), denominada ahora $A_{C}$, resulta:

$A_{C}=A-B R^{-1} B^{T} \tilde{P}$ 
La matriz $\widetilde{P}$ se obtiene resolviendo la siguiente ecuación matricial de Riccati

$\left(A+h_{1} I_{n}\right)^{T} \widetilde{P}+\widetilde{P}\left(A+h_{1} I_{n}\right)-\widetilde{P} B R^{-1} B^{T} \widetilde{P}=0$

en la cual puede verse que no interviene la matriz de peso $Q$.

La acción de control instrumentada con las ecuaciones (12) a (14) ha logrado modificar las componentes reales de los autovalores $\lambda_{l}$ a $\lambda_{m}$ del sistema eléctrico a lazo abierto (matriz A en (6)) llevándolas a posiciones ubicadas a la izquierda de la línea vertical $\left(-h_{1}\right)$ indicada en la Figura 2a. Sin embargo, como existen restricciones físicas sobre los parámetros reales (constantes de tiempo y ganancias), debe limitarse la ubicación de los autovalores $\lambda_{1}$ a $\lambda_{1}$, a una región definida como la indicada en la Figura $2 b$.

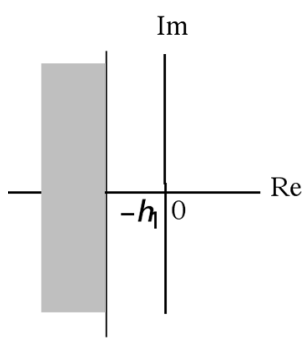

(a) Plano vertical

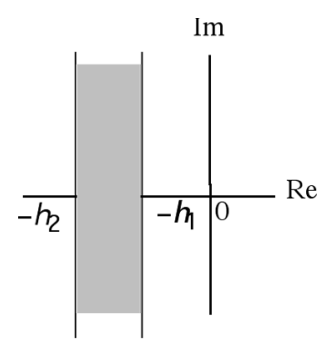

(b) Franja vertical
Figura 2. Plano complejo s.

Suponiendo que $h_{1}$ y $h_{2}$ son dos valores reales positivos $\left(h_{2}>h_{1}\right)$ que definen una franja vertical $\left(-h_{2},-h_{1}\right)$ de espesor $\left(h_{2}-h_{1}\right)$ según se muestra en la Figura 2b. y siendo la matriz $\tilde{A}=A+h_{1} I_{n}$, entonces para lograr este propósito, la ley de control debe modificarse a (Shieh et al., 1986):

$$
\Delta U=-\rho \quad \tilde{F} \Delta X
$$

en donde la ganancia del lazo cerrado de retroalimentación $\widetilde{F}$ surge de (12) y la matriz $\widetilde{P}$ es la solución de la ecuación de Riccati modificada ,que coincide con la ecuación (14).
$\widetilde{A}^{T} \widetilde{P}+\widetilde{P} \widetilde{A}-\widetilde{P} B R^{-1} B^{T} \widetilde{P}=0$

Según (Shieh et al., 1986) la ganancia $\rho$ se selecciona con la siguiente expresión:

$\rho=\frac{1}{2}+\left(h_{2}-h_{1}\right) / 2 \operatorname{tr}(\tilde{A})=\frac{1}{2}+\left(h_{2}-h_{1}\right) / 2 \operatorname{tr}(B \tilde{F})$

siendo

$\operatorname{tr}(\widetilde{A})=\sum^{m} \lambda_{i}=\frac{1}{2} \operatorname{tr}(\tilde{B F})$ y $\lambda_{i}(i=1, \ldots, m)$ los autovalores (complejos conjugados) de $\tilde{A}$ ubicados en el semiplano derecho del plano $s$. De este modo, el sistema óptimo de lazo cerrado es, en consecuencia:

$\Delta \dot{X}=(A-\rho B \widetilde{F}) \Delta X$

Esto facilita la acción de coordinación de los dispositivos PSS de ser ajustados para mejorar el amortiguamiento de las OP.

\section{PRUEBA CON UN SISTEMA LONGITUDINAL}

Para llevar a cabo una serie de pruebas con un sistema eléctrico de tamaño considerable, con el fin de observar la contribución de los PSS sobre el amortiguamiento de las OP, es necesario tener presente que éstas se manifiestan según la estructura topológica de la red, las condiciones de operación y los efectos de los dispositivos de control. En vista de lo anterior y con base en el análisis efectuado, se utilizará como modelo de prueba la red de alta tensión en doscientos treinta mil voltios, del Sistema Interconectado Centroamericano (SICA), por cuanto este sistema presenta una estructura longitudinal y se cataloga como un sistema débilmente interconectado.

El SICA será analizado en dos subsistemas. El norte, constituido por Guatemala y El Salvador, y el sur, compuesto por Honduras, Nicaragua, Costa Rica y Panamá. En este análisis se efectuó la coordinación de los PSS para cada subsistema y para el sistema del Istmo en conjunto, tomando en cuenta la interconexión entre Honduras y El Salvador efectuada en el año 2002. En los Cuadros 1, 2 y 3 se resumen los resultados obtenidos. 
Cuadro 1. Principales modos de oscilación electromecánicos, para el esquema de generación de demanda mínima en invierno, con intercambiode potencia de 100 MW de Guatemala a El Salvador.

$\begin{array}{lccc}\text { Modo } & \text { Frecuencia }(\mathrm{Hz}) & \text { Sin PSS } & \text { PSS coord. } \\ & & & \\ 1 & 0,760 & -0,037 \pm \mathrm{j} 4,963 & -1,262 \pm \mathrm{j} 4,925 \\ 2 & 0,861 & -0,016 \pm \mathrm{j} 5,426 & -1,091 \pm \mathrm{j} 5,427 \\ 3 & 1,169 & -0,003 \pm \mathrm{j} 7,510 & -1,891 \pm \mathrm{j} 7,505 \\ 4 & 1,370 & 0,076 \pm \mathrm{j} 8,513 & -1,851 \pm \mathrm{j} 8,424 \\ 5 & 1,374 & 0,123 \pm \mathrm{j} 8,999 & -1,837 \pm \mathrm{j} 8,846\end{array}$

Cuadro 2. Principales modos de oscilación electromecánicos, para el esquema de generación de demanda mínima en invierno, con intercambio de potencia de 100 MW de Honduras a Panamá.

$\begin{array}{lccc}\text { Modo } & \text { Frecuencia }(\mathrm{Hz}) & \text { Sin PSS } & \text { PSS coord. } \\ 1 & 0,648 & -0,062 \pm \mathrm{j} 3,471 & -1,752 \pm \mathrm{j} 3,673 \\ 2 & 0,997 & -0,045 \pm \mathrm{j} 6,235 & -1,245 \pm \mathrm{j} 6,222 \\ 3 & 1,224 & -0,300 \pm \mathrm{j} 7,672 & -1,620 \pm \mathrm{j} 7,653 \\ 4 & 1,282 & -0,086 \pm \mathrm{j} 8,146 & -1,453 \pm \mathrm{j} 8,289 \\ 5 & 1,352 & -0,007 \pm \mathrm{j} 8,450 & -1,314 \pm \mathrm{j} 8,258 \\ 6 & 1,576 & 0,017 \pm \mathrm{j} 10,360 & -1,017 \pm \mathrm{j} 10,325\end{array}$

Cuadro 3. Principales modos de oscilación electromecánicos, para el esquema de generación de demanda mínima en invierno, con intercambio de potencia de 100 MW de Guatemala a Panamá.

$\begin{array}{lccc}\text { Modo } & \text { Frecuencia (Hz) } & \text { Sin PSS } & \text { PSS coord. } \\ 1 & 0,494 & -0,017 \pm \mathrm{j} 3,102 & -1,409 \pm \mathrm{j} 2,981 \\ 2 & 0,806 & -0,043 \pm \mathrm{j} 5,062 & -1,513 \pm \mathrm{j} 4,998 \\ 3 & 0,958 & -0,055 \pm \mathrm{j} 6,017 & -1,881 \pm \mathrm{j} 5,993 \\ 4 & 1,061 & 0,060 \pm \mathrm{j} 6,667 & -1,509 \pm \mathrm{j} 6,651 \\ 5 & 1,149 & -0,159 \pm \mathrm{j} 7,217 & -1,265 \pm \mathrm{j} 7,223\end{array}$

En esta etapa del análisis se pone de manifiesto el efecto del tipo de regulador de tensión (giratorio, estático) y el efecto de la acción coordinada de los dispositivos PSS. En los cuadros anteriores, los autovalores obtenidos a través de la coordinación de los PSS, presentan un mejor rendimiento en cuanto a su amortiguamiento relativo, manteniendo aproximadamente constante la frecuencia de oscilación.

\section{CONCLUSIONES}

En vista de los resultados anteriores, con el PSS en servicio, las conclusiones son:

a. En la propuesta contenida en este trabajo se ha elegido también a la matriz $R$ como matriz identidad, con lo cual los $m$ elementos de control participan en forma independiente unos de otros y con igual peso en la acción de control a realizar. En cuanto a la matriz $Q$ se ha prescindido de ella y, en su lugar, se ha empleado un método de traslación de autovalores que afecta solamente a aquellos autovalores cuyas partes reales son positivas. El efecto es tal, que modifica solamente las partes reales de los autovalores, manteniendo aproximadamente constantes las respectivas partes imaginarias. Las nuevas partes reales de los autovalores, por efecto de la traslación lograda, quedan ubicadas en el semiplano izquierdo del plano complejo y dentro de una franja vertical de ancho especificado $a$ priori.

b. La efectividad de esta propuesta de coordinación de las acciones de control de los PSS para mejorar el amortiguamiento de las OP ha sido constatada mediante cálculo en el Sistema Eléctrico Interconectado Centroamericano (SICA). En todos los casos, se obtuvo un mejoramiento en los modos de oscilación, que inicialmente eran pobremente amortiguados.

c. Al implementar esta propuesta en programas de cálculo digital, los diferentes componentes dinámicos del sistema eléctrico han sido incluidos en forma modular. Esto constituye una ventaja, pues permite, en caso de ser necesario, actualizar cómodamente los modelos del generador, del regulador automático de tensión y de los dispositivos PSS. Además, la inclusión de nuevos componente dinámicos tales como los dispositivos FACTS (Flexible ac Transmission Systems) resultaría muy sencilla. 


\section{SIMBOLOGÍA}

D coeficiente de amortiguamiento

$H \quad$ constante de inercia

$M=2 H$ Constante de inercia modificada

$\mathrm{s}=\mathrm{j} \omega$ operador de Laplace

$T_{e} \quad$ par eléctrico

$T_{m} \quad$ par mecánico

$T_{s} \cdot \Delta \delta \quad$ cambio en el par sincronizante

$T_{D} \cdot \Delta \omega$ cambio en el par de amortiguamiento

$\delta \quad$ ángulo entre el eje en cuadratura y la barra de potencia infinita

$\omega \quad$ velocidad angular del rotor del generador, alrededor del punto de operación

$\Delta \quad$ subíndice que indica un pequeño cambio en alguna variable

\section{BIBLIOGRAFÍA}

Quesada, R. (1986). Estudio de estabilidad dinámica en el dominio de la frecuencia para el Sistema Interconectado Costa RicaPanamá. Tesis de Maestría, DIPFI, UNAM, México.

Sancho, J. (1998). Metodología para realizar estudios de seguridad operativa en el Sistema Interconectado Centroamericano. Tesis de Maestría, Escuela de Ingeniería Eléctrica, Universidad de Costa Rica, San José, Costa Rica.

Molina, D. (1997, mayo). Diagnóstico de la estabilidad oscilatoria del SADI - Criterios Prácticos - Análisis Modal - Estudios y Equipos Complementarios. En: CIGRE, VII Encuentro Regional Latinoamericano, Puerto Iguazú, Argentina.

Messina, R. (1995). Experience with the analysis of small signal stability in longitudinal systems: a case study with the Mexican Interconnected System. Electric Power \& Energy Systems, 17(5), 291-299.

Ogata, K. (1993). Ingeniería de control moderna. Prentice-Hall.

Laub, A. J. (1979). A Schur method for solving algebraic Riccati equations. IEEE Trans. on Automatic Control, December, AC-24(6), 913-921.

Moussa, A. M. \& Yu, Y. (1972). Optimal power systems stabilization through excitation and/ or governor control. IEEE IEEE Transaction on PAS, May/June, 1166-1174.

Yu, Y. \& Moussa, A. M. (1972). Optimal stabilization of a multi-machine systems. IEEE Transaction on PAS, May/June, 11741182.

Kawasaki, N. \& Shimemura, E. (1983). Determining quadratic weighting matrices to locate poles in a specified region. Automatica, 19(5), 557-560.

Shieh, L. S., Dib, H. M., \& Mcinnis, B. C. (1986, Mar). Linear quadratic regulators with eigenvalue placement in a vertical strip. IEEE Trans. on Automatic Control, AC31(3), 241-243.

\section{SOBRE EL AUTOR}

\section{Eddie A. Araya Padilla}

Ingeniero electricista, $\mathrm{Ph}, \mathrm{D}$.; profesor del Departamento de Sistemas de Potencia de la Escuela de Ingeniería Eléctrica de la Universidad de Costa Rica

Teléfonos: 207-4329 ó 207-5146

Facsímil: 207-4139

Correo electrónico: earaya@eie.ucr.ac.cr 
